\title{
Ten years risk assessment of atherosclerotic cardiovascular disease using Astro-CHARM and pooled cohort equation in a south Asian sub-population
}

\author{
Tariq Ashraf \\ National Institute of Cardiovascular Diseases (NICVD), Karachi, Pakistan \\ Muhammad Naeem Mengal \\ National Institute of Cardiovascular Diseases (NICVD), Pakistan \\ Atif Sher Muhammad \\ National Institute of Cardiovascular Diseases (NICVD), Karachi, Pakistan \\ Asal Khan Tareen \\ National Institute of Cardiovascular Diseases (NICVD), Karachi, Pakistan \\ Nauman Khan \\ National Institute of Cardiovascular Diseases (NICVD), Karachi, Pakistan
}

See next page for additional authors

Follow this and additional works at: https://ecommons.aku.edu/pakistan_fhs_mc_mc

Part of the Cardiovascular Diseases Commons, and the Public Health Commons

\section{Recommended Citation}

Ashraf, T., Mengal, M. N., Muhammad, A. S., Tareen, A. K., Khan, N., Kazmi, K. A., Nadeem, A., Sarwar, S., Bashir, Z., Qamar, N., Karim, M. (2020). Ten years risk assessment of atherosclerotic cardiovascular disease using Astro-CHARM and pooled cohort equation in a south Asian sub-population. BMC Public Health, 20(1), 403.

Available at: https://ecommons.aku.edu/pakistan_fhs_mc_mc/115 


\section{Authors}

Tariq Ashraf, Muhammad Naeem Mengal, Atif Sher Muhammad, Asal Khan Tareen, Nauman Khan, Khawar Abbas Kazmi, Asif Nadeem, Shakil Sarwar, Zara Bashir, Nadeem Qamar, and Musa Karim 


\title{
Ten years risk assessment of atherosclerotic cardiovascular disease using Astro-CHARM and pooled cohort equation in a south Asian sub-population
}

Tariq Ashraf ${ }^{*}$, Muhammad Naeem Mengal ${ }^{1}$, Atif Sher Muhammad ${ }^{1}$, Asal Khan Tareen', Muhammad Nauman Khan ${ }^{1}$, Khawar Abbas Kazmi ${ }^{1}$, Asif Nadeem², Shakil Sarwar², Zara Bashir ${ }^{3}$, Nadeem Qamar ${ }^{1}$ and Musa Karim ${ }^{1}$

\begin{abstract}
Background: Atherosclerotic cardiovascular diseases (ASCVD) are on the rise in low and middle-income countries attributed to modern sedentary lifestyle and dietary habits. This has led to the need of assessment of the burden of at-risk population so that prevention measures can be developed. The objective of this study was to assess ten years risk assessment of ASCVD using Astro-CHARM and Pooled Cohort Equation (PCE) in a South Asian subpopulation.

Methods: A total of 386 residents of all six districts of Karachi with no ASCVD were enrolled in the study through an exponential non-discriminative referral snowball sampling technique. The inclusion criteria consisted of age 40 years or above and either gender. Study participants were enrolled after obtaining informed written consent and those study participants who were found to have either congenital heart disease or valvular heart diseases or ischemic heart disease were excluded from the study based on initial screening. For the calculation of 10 years risk of ACVD based on Astro-CHARM and PCE, the variables were obtained including medical history and coronary artery calcium and C-reactive protein measurements.

Results: Mean estimated 10-year risk of fatal or non-fatal myocardial infarction or stroke as per the Astro-CHARM was $13.98 \pm 8.01 \%$, while mean estimated 10-year risk of fatal or non-fatal myocardial infarction or stroke as per the PCE was $22.26 \pm 14.01 \%$. Based on Astro-CHARM, 11.14\% of the study participants were labeled as having high risk, while PCE estimated $20.73 \%$ of study participants as having high risk of ASCVD.

Conclusion: Despite the fact that our findings showed substantial differences in ten-year risk of ASCVD between Astro-CHARM and PCE, both calculators can be used to develop a new population and specific risk estimators for this South Asian sub-population. Our study provides the first step towards developing a risk assessment guided decision-making protocol for primary prevention of ASCVD in this population.
\end{abstract}

Keywords: Atherosclerotic cardiovascular disease, Astro-CHARM, Pooled cohort equation, South Asia

\footnotetext{
* Correspondence: tariqash45@gmail.com

1 National Institute of Cardiovascular Diseases (NICVD), Karachi, Pakistan

Full list of author information is available at the end of the article
}

(c) The Author(s). 2020 Open Access This article is licensed under a Creative Commons Attribution 4.0 International License, which permits use, sharing, adaptation, distribution and reproduction in any medium or format, as long as you give appropriate credit to the original author(s) and the source, provide a link to the Creative Commons licence, and indicate if changes were made. The images or other third party material in this article are included in the article's Creative Commons licence, unless indicated otherwise in a credit line to the material. If material is not included in the article's Creative Commons licence and your intended use is not permitted by statutory regulation or exceeds the permitted use, you will need to obtain permission directly from the copyright holder. To view a copy of this licence, visit http://creativecommons.org/licenses/by/4.0/ The Creative Commons Public Domain Dedication waiver (http://creativecommons.org/publicdomain/zero/1.0/) applies to the data made available in this article, unless otherwise stated in a credit line to the data. 


\section{Background}

With the ever-increasing global burden of atherosclerotic cardiovascular diseases (ACVD) the need to timely assess the risk of cardiovascular events cannot be more emphasized [1-5]. Once among the most common causes of death in high-income countries only, ACVD has become the leading cause of morbidity and mortality in low and middle-income countries as well owing to epidemiologic transition and changing population dynamics. This trend is more prominent in the South Asian population where (ACVD) is the leading cause of death $[6,7]$.

A number of risk estimators have been developed to assess the risk of any cardiovascular event in at-risk population. Astro-CHARM and Pooled Cohort Equation (PCE) has been much widely used worldwide however these estimators have been developed in the west and not much evidence is present that how accurately they estimate ACVD in South Asian population [8, 9]. Tariq et al. [10] reported that the Pakistani population showed to have a higher risk of ASCVD compared to whites, blacks, and Hispanic and even South Asians living in USA. Another study conducted in a similar population showed that the burden of premature myocardial infarction to be as high as 12\% [11]. In addition, another large multi-centric study from Pakistan attempted to predict the risk of atherosclerotic cardiovascular disease in Pakistani population, however, included subject with already suffered from ASCVD [12]. Such methodological flaws can have serious implications while projecting risk assessment on the general population. There is a scarcity of literature providing estimates of ASCVD risk in resident South Asian population as well as comparison of different risk estimators in this population.

Majority of these studies have used PCE as a risk estimator which has its own limitation that includes overestimating the risk in other nationalities/ ethnicities and in population with less social deformation which requires attention while making medical decisions. To overcome this Astro-CHARM calculation was used (Astronaut Cardiovascular Health and Risk Modification). Data in white, Hispanic \& black have been elucidated except for the South Asians [8].

Cardiovascular risk can be estimated as either absolute, relative, lifetime, or recurrent. An internationally agreed-upon guidelines do not exist in this regard [13]. The 10 years risk of coronary events (high, intermediate, or low) can be determined by various risk scores, such as laboratory and non-laboratory Framingham risk score for CVD, systematic coronary risk evaluation, Reynolds risk score, the Lancet chronic diseases risk charts, World Health Organization risk charts, etc., but poor concordance has been reported between the risk score, jeopardizing their clinical utility [14]. ACC/AHA preventive guidelines advocate the use of PCE for the assessment of cardiovascular risk [15] and Astro-CHARM is considered to be a significant improvement over the PCE with the addition of discriminating power of coronary artery calcium (CAC) and hs-CRP [8]. Keeping in mind the ease of adoption of PCE in a resource-limited setting and predictive superiority of Astro-CHARM, we assessed the risk of ASCVD in our population using both modalities.

This study, to the best of our knowledge, attempted to estimate the ten-year risk of ASCVD in general population without any history of ASCVD using both AstroCHARM and PCE, the aim of this study was to estimate risk and compared both the modalities.

\section{Methods}

This analytical cross-sectional study was conducted among 386 residents of all six districts of Karachi who had no prior history of atherosclerotic cardiovascular disease including stroke. Study was approved by the ethical review committee of the National Institute of Cardiovascular Diseases (NICVD) Karachi, Pakistan (ERC-29/2018). Karachi is the largest metropolitan city of the country with a diverse ethnic distribution, inhabiting around 15 million people with a fair share of all the ethnicities residing in the country. Due to non-availability of the sampling frame for any probability sampling technique, non-probability snowball sampling technique was adopted. Starting with the recruitment of consenting individuals, for all six districts of the city, meeting the inclusion criteria of the study from healthy attendants visiting our center. The exponential non-discriminative referral technique was adopter where every recruited participant was allowed multiple referrals from his/her acquaintances. All the referrals were contacted and directed to a designated desk at the doctor's office for initial screening and assessment. After a thorough history, physical, and electrocardiographic assessment to rule out underlying atherosclerotic cardiovascular disease, participants of both gender and age between 40 to 65 years were included in this study. Study participants were enrolled after obtaining informed written consent and those study participants who were found to have either congenital heart disease or valvular heart diseases or ischemic heart disease were excluded from the study based on initial screening. Individuals who refused or shown reluctance to accept computed tomography angiography (CTA) or other laboratory assessments such as lipid profile or C-reactive protein (hs-CRP) were excluded after an initial assessment.

Fasting (12 h) lipid profile and hs-CRP level were obtained for all the enrolled patients and for the calculation of 10 years risk of ACVD based on Astro-CHARM and PCE, the variables were obtained in the following manner. 
Diabetes mellitus, hypertension, current smoking, and family history of heart attack were defined as per AstroCHARM definitions [8].

High-sensitivity C-reactive protein (hs-CRP) levels were measured by using the following assays. MESA, BNII nephelometer ( $\mathrm{N}$ High Sensitivity CRP; Dade Behring Inc); DHS, Roche/Hitachi 912 System, Tina-quant assay (Roche Diagnostics), alatex-enhanced immunoturbidimetric method; PACC, particleenhancedimmune turbidimetric latex agglutination assay; FHS, enhanced immune turbidimetric high-sensitivity assay (Roche Diagnostics). Computed tomography angiography (CTA) was performed in all the patients and coronary artery calcium (CAC) score was calculated for all the patients.

Data were entered using MS Excel for Windows and analyses were done using STATA 11.0 while 10 years risk of ACVD was calculated using Astro-CHARM and PCE using the online calculators available at www.AstroCHARM.org and www.clincalc.com respectively. Participants were stratified as low-risk and high-risk individuals based on cut-off have of $\geq 7.5 \%$ for both Astro-CHARM and PCE.

Descriptive analyses were conducted by calculating the mean and standard deviation for all continuous variables while frequency and percentages were calculated for all categorical variables. Comparison between male and female participants on continuous variables was made by performing independent sample t-test and categorical variables were compared by applying Chi-square test and $p$-value $\leq 0.05$ as taken as criteria for statistical significance. Risk of ACVD was reported using both equations for the combined study population and was also stratified for different sub-groups.

\section{Results}

Mean age of the study participants was $49 \pm 7.10$ years with $54 \%$ Males. Mean BMI was $28.11 \pm 5.38$ while 45.6 , 15.8 , and $14.2 \%$ were hypertensive, diabetics and current smokers respectively. Six (2\%) of the study participants had all three co-morbid conditions. Thirty percent had positive family history of MI. Mean CAC was $7.50 \pm$ 14.05 Agatston units. Table 1 shows the baseline sociodemographic and medical characteristics of study participants stratified by gender.

Mean estimated 10-year risk of fatal or non-fatal myocardial infarction or stroke as per the Astro-CHARM was $13.98 \pm 8.01 \%$, while mean estimated 10 -year risk of fatal or non-fatal myocardial infarction or stroke as per the PCE was $22.26 \pm 14.01 \%$. Risk comparison in different age categories showed a significantly higher risk of ACVD in age groups of 55-60 years and 60 years and above compared to the ASCVD risk in the age group 40-45 years as shown in Fig. 1.

Similarly, Table 2 below shows the comparison of baseline characteristics of high and low-risk individuals

Table 1 Baseline socio-demographic and medical characteristics of study participants

\begin{tabular}{|c|c|c|c|c|}
\hline \multirow[t]{2}{*}{ Baseline characteristics } & \multirow{2}{*}{$\begin{array}{l}\text { Overall } \\
\boldsymbol{N}=386\end{array}$} & \multicolumn{2}{|l|}{ Gender } & \multirow[t]{2}{*}{$\boldsymbol{P}$-value } \\
\hline & & $\begin{array}{l}\text { Male } \\
\boldsymbol{N}=211\end{array}$ & $\begin{array}{l}\text { Female } \\
\boldsymbol{N}=175\end{array}$ & \\
\hline Age (years) ${ }^{a}$ & $49 \pm 7.11$ & $49.47 \pm 7.24$ & $48.43 \pm 6.93$ & 0.153 \\
\hline Body mass index $\left(\mathrm{kg} / \mathrm{m}^{2}\right)^{\mathrm{a}}$ & $28.12 \pm 5.39$ & $26.93 \pm 4.71$ & $29.55 \pm 5.8$ & $<0.001$ \\
\hline Coronary artery calcium (Agatston units) ${ }^{a}$ & $31.57 \pm 195.63$ & $44.61 \pm 253.95$ & $15.86 \pm 79.94$ & 0.121 \\
\hline Total cholesterol $(\mathrm{mg} / \mathrm{dL})^{\mathrm{a}}$ & $177.65 \pm 38.97$ & $174.91 \pm 37.02$ & $180.94 \pm 41.07$ & 0.131 \\
\hline HDL cholesterol $(\mathrm{mg} / \mathrm{dL})^{\mathrm{a}}$ & $51.73 \pm 36.78$ & $50.93 \pm 48.39$ & $52.7 \pm 12.85$ & 0.639 \\
\hline Systolic blood pressure $(\mathrm{mmHg})^{a}$ & $138.83 \pm 21.23$ & $135.73 \pm 20.81$ & $142.57 \pm 21.18$ & 0.002 \\
\hline \multicolumn{5}{|l|}{ Hypertensive } \\
\hline Yes & $176(45.6 \%)$ & $78(36.97 \%)$ & $98(56 \%)$ & \multirow[t]{2}{*}{$<0.001$} \\
\hline No & $210(54.4 \%)$ & $133(63.03 \%)$ & 77 (44\%) & \\
\hline \multicolumn{5}{|l|}{ Diabetic } \\
\hline Yes & $61(15.8 \%)$ & $34(16.11 \%)$ & $27(15.43 \%)$ & \multirow[t]{2}{*}{0.854} \\
\hline No & $325(84.2 \%)$ & 177 (83.89\%) & $148(84.57 \%)$ & \\
\hline \multicolumn{5}{|l|}{ Current Smoker } \\
\hline Yes & $55(14.25 \%)$ & $50(23.7 \%)$ & $5(2.86 \%)$ & \multirow[t]{2}{*}{$<0.001$} \\
\hline No & $331(85.75 \%)$ & $161(76.3 \%)$ & 170 (97.14\%) & \\
\hline \multicolumn{5}{|l|}{ Family History of Myocardial Infarction } \\
\hline Yes & 118 (30.57\%) & $66(31.28 \%)$ & $52(29.71 \%)$ & \multirow[t]{2}{*}{0.74} \\
\hline No & $268(69.43 \%)$ & $145(68.72 \%)$ & $123(70.29 \%)$ & \\
\hline
\end{tabular}

a reported as mean and standard deviation, all other variables are reported as frequencies and percentages 


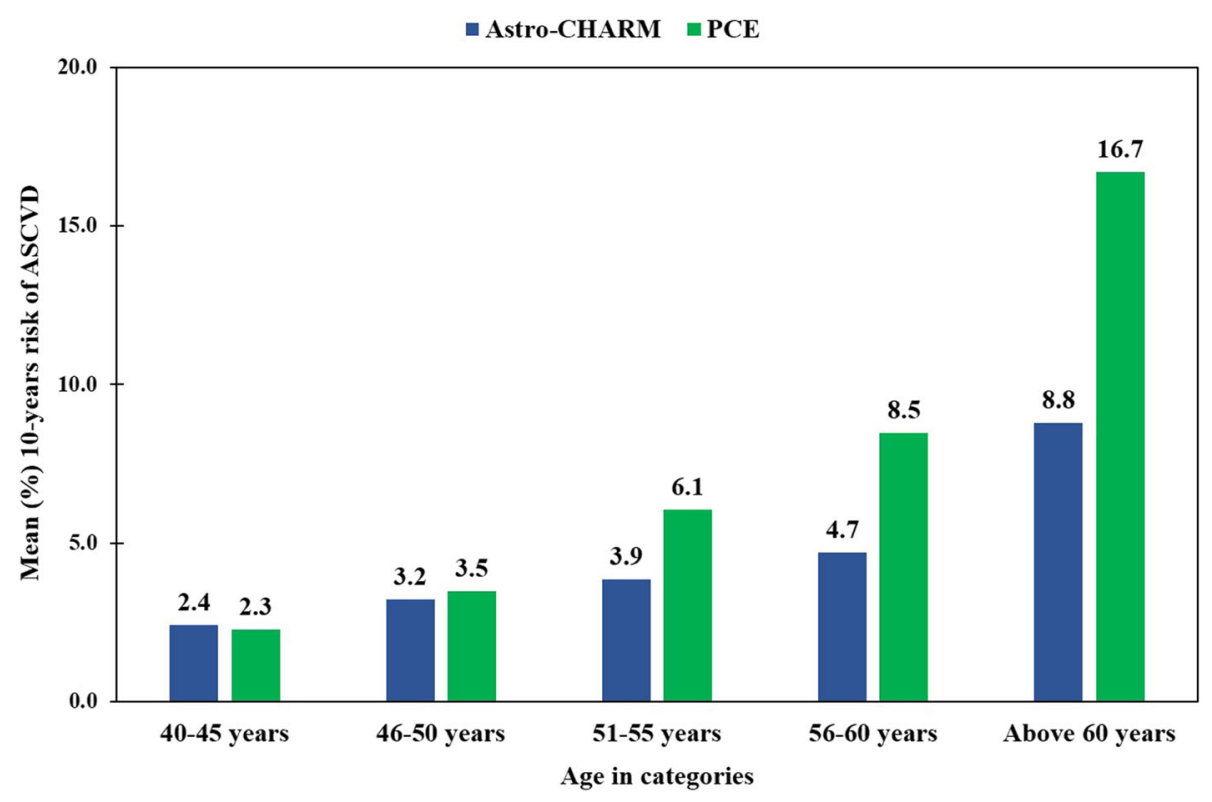

Fig. 1 The comparison of mean 10-years risk of ASCVD for different age groups as calcualted by Astro-CHARM and PCE

based on Astro-CHARM and Pooled Cohort Estimator. Kappa agreement between the two calculators in terms of labeling high and low-risk individuals was high (0.50, $p$-value $<0.0001)$.

Out of 386 individuals, $8.8 \%$ (34) were categorized as high risk by both Astro-CHARM and PCE and 76.9\% (297) were categorized as low risk by both AstroCHARM and PCE. Taking Astro-CHARM as gold standard for categorizing high risk individuals, PCE has sensitivity of $79.07 \%$, specificity of $86.59 \%$, positive predictive value of $42.50 \%$, and negative predictive value of $97.06 \%$, as presented in Table 3 .

\section{Discussion}

To the best of our knowledge, current study reports 10 year risk of ASCVD among non-cardiac Pakistani population using both Astro-CHARM and PCE and the comparison of the two calculators for the first time. In concordance with the findings of other studies, PCE estimated the risk of ASCVD in the study population almost twice as that estimated by Astro-CHARM. AstroCHARM which also includes indicators like coronary artery calcium and C-reactive protein during ASCVD risk assessment, these indicators are not readily available in low and middle-income countries, therefore, the significance of PCE cannot be neglected [16].

One of the key findings of this study was that both coronary artery calcium and $\mathrm{C}$ reactive protein significantly correlated with the risk scores generated by PCE which is in concordance with findings from other studies
$[17,18]$. Especially in the case of CAC, a relatively recent study by JJ Carr et al. [17] reported its effect on the risk of ASCVD among individuals well below 40 years of age. Keeping in mind that said study was conducted among African-Americans and Caucasians, who are relatively at lower risk of developing ASCVD compare to South Asians, it points towards a major limitation of these risk calculators among South Asians, who, in clinical experience suffer cardiac events at an even younger age [19] and evidenced by the previous study from Pakistan [10], there is a dire need of development of populationspecific risk estimator for the South Asian population. This study provided the grounds towards developing such risk estimator as well as initial step towards developing evidence-based decision making for primary prevention guidelines of atherosclerotic cardiovascular disease in this population.

Considering the fact that the population of South Asian countries consists of multi-ethnical groups with possibly different inherent risk of developing ASVD, as well as the absence of risk estimators in population below 40 years of age much evidence is required to develop population-specific risk estimators and risk guided decision-making tools for primary prevention among South Asians using robust study designs. Furthermore, considering the sub-optimal healthcare systems and the absence of expensive investigating facilities like CAC in low and middle-income countries of South Asia, the development of risk estimators for these populations also requires large population-based, ethnicity and 
Table 2 Comparison of baseline characteristics of high and low risk individuals based on Astro-CHARM and Pooled Cohort Estimator

\begin{tabular}{|c|c|c|c|c|}
\hline \multirow[t]{2}{*}{ Characteristics } & \multicolumn{2}{|l|}{ Astro-CHARM } & \multicolumn{2}{|l|}{ PCE } \\
\hline & $\begin{array}{l}\text { Low Risk } \\
N=343\end{array}$ & $\begin{array}{l}\text { High Risk } \\
N=43\end{array}$ & $\begin{array}{l}\text { Low Risk } \\
N=306\end{array}$ & $\begin{array}{l}\text { High Risk } \\
N=80\end{array}$ \\
\hline \multicolumn{5}{|l|}{ Gender } \\
\hline Male & $51.9 \%(178)$ & $76.7 \%$ (33) & $47.4 \%(145)$ & $82.5 \%(66)$ \\
\hline Female & $48.1 \%(165)$ & $23.3 \%(10)$ & $52.6 \%(161)$ & $17.5 \%(14)$ \\
\hline Weight (kg) & $73 \pm 15$ & $74 \pm 14$ & $74 \pm 15$ & $72 \pm 13$ \\
\hline Height (cm) & $162 \pm 11$ & $165 \pm 10$ & $161 \pm 11$ & $166 \pm 10$ \\
\hline Age (years) & $48 \pm 7$ & $55 \pm 7$ & $47 \pm 6$ & $57 \pm 6$ \\
\hline \multicolumn{5}{|l|}{ Hypertensive } \\
\hline Yes & $44.6 \%(153)$ & $53.5 \%(23)$ & $44.4 \%(136)$ & $50 \%(40)$ \\
\hline No & $55.4 \%(190)$ & $46.5 \%(20)$ & $55.6 \%(170)$ & $50 \%(40)$ \\
\hline \multicolumn{5}{|l|}{ Diabetic } \\
\hline Yes & $11.1 \%(38)$ & $53.5 \%(23)$ & $8.8 \%(27)$ & $42.5 \%(34)$ \\
\hline No & $88.9 \%$ (305) & $46.5 \%(20)$ & $91.2 \%$ (279) & $57.5 \%(46)$ \\
\hline \multicolumn{5}{|l|}{ Current Smoker } \\
\hline Yes & $11.1 \%(38)$ & $39.5 \%(17)$ & $9.2 \%(28)$ & $33.8 \%(27)$ \\
\hline No & $88.9 \%$ (305) & $60.5 \%(26)$ & $90.8 \%$ (278) & $66.2 \%(53)$ \\
\hline \multicolumn{5}{|l|}{ Family History of Myocardial infarction } \\
\hline Yes & $28.6 \%(98)$ & $46.5 \%(20)$ & $32 \%(98)$ & $25 \%(20)$ \\
\hline No & $71.4 \%(245)$ & $53.5 \%(23)$ & $68 \%(208)$ & $75 \%(60)$ \\
\hline Coronary Artery Calcium (Agatston) & $9.9 \pm 46.42$ & $204.2 \pm 546.76$ & $15.5 \pm 73.87$ & $93 \pm 400.79$ \\
\hline Total Cholesterol (mg/dL) & $178 \pm 37$ & $178 \pm 50$ & $175 \pm 39$ & $187 \pm 37$ \\
\hline HDL Cholesterol (mg/dL) & $53 \pm 39$ & $45 \pm 10$ & $53 \pm 41$ & $45 \pm 11$ \\
\hline Systolic Blood Pressure (mmHg) & $137 \pm 20$ & $155 \pm 21$ & $137 \pm 20$ & $148 \pm 22$ \\
\hline
\end{tabular}

Astro-CHARM Astronaut Cardiovascular Health and Risk Modification; PCE Pooled Cohort Equation Low-Risk $=<7.5 \%$ estimated 10 years risk; High-Risk $=\geq 7.5 \%$ estimated 10 years risk

age-specific data to develop such a tool using both Astro-CHARM and PCE. Low and middle-income countries are now overburdened with interventional procedures like angioplasty, and expensive methods of secondary prevention. Accurate risk assessment is the only solution in such resource-poor settings where

Table 3 Cross tabulation of Pooled Cohort Estimator against Astro-CHARM for categorizing high risk individuals

\begin{tabular}{lll}
\hline & \multicolumn{2}{l}{ Astro-CHARM } \\
\cline { 2 - 2 } & Low Risk & High Risk \\
\hline PCE & $76.9 \%(297)$ & $2.3 \%(9)$ \\
How Risk & $11.9 \%(46)$ & $8.8 \%(34)$ \\
Sensitivity & $79.07 \%(95 \% \mathrm{Cl}: 63.96$ to $89.96 \%)$ \\
Specificity & $86.59 \%(95 \% \mathrm{Cl}: 82.52$ to $90.01 \%)$ \\
Positive predictive value & $42.50 \%(95 \% \mathrm{Cl}: 35.16$ to $50.19 \%)$ \\
Negative predictive value & $97.06 \%(95 \% \mathrm{Cl}: 94.85$ to $98.34 \%)$
\end{tabular}

Astro-CHARM Astronaut Cardiovascular Health and Risk Modification, PCE Pooled Cohort Equation, $\mathrm{Cl}$ Confidence interval

Low-Risk $=<7.5 \%$ estimated 10 years risk; High-Risk $=\geq 7.5 \%$ estimated 10 years risk primary prevention can be implicated through lifestyle modifications and statins among high-risk individuals. However, overestimation and misclassification of an individual as high risk has its own implications too, not only at the individual level but also at healthcare system level, therefore, accuracy of the risk stratification modality is crucial.

\section{Strengths and limitations}

The biggest strength of the study is the use and comparison of both ASCVD estimators in non-cardiac Pakistani general population selected from the largest city of the country with diverse population sub-groups from the entire country thus providing data facsimile to general population of Pakistan.

Major limitations of the study lie in the nonprobability snowball sampling technique and study design where lack of prospective follow-up cannot ensure the accuracy of these estimates. Furthermore, the data was taken from an urban setting and implications of the findings cannot be ascertained on the rural population. 


\section{Conclusion}

Despite the fact that our findings showed substantial differences in 10-year risk of ASCVD between AstroCHARM and PCE, both calculators can be used to develop a new population and specific risk estimators for this South Asian sub-population. Our study provides the first step towards developing a risk assessment guided decision-making protocol for primary prevention of ASCVD in this population.

\section{Abbreviations}

ASCVD: Atherosclerotic cardiovascular diseases; Astro-CHARM: Astronaut cardiovascular health and risk modification; CAC: Coronary artery calcium; CTA: Computed tomography angiography; CVD: Cardiovascular disease; hsCRP: High sensitive C-reactive protein; NICVD: National institute of cardiovascular diseases; PCE: Pooled cohort equation; USA: United Sates of America

\section{Acknowledgments}

Authors are thankful to the Medical Affairs division of Getz Pharma for providing financial support for $C T$ angiography and lab assessments of the study participants. The authors wish to acknowledge the support of the staff member of the clinical research department of the National Institute of Cardiovascular Diseases (NICVD), Karachi Pakistan.

\section{Authors' contributions}

TA conceptualized the study and supervised the entire project, MNM, ASM, AKT, MNK and KAK conducted the study and data collection from patients, AN and SS conducted the data collection and facilitated CT angiography, ZB provided technical support while NQ and MK conducted the statistical analyses. All authors were involved and contributed at each step of manuscript writing. The authors read and approved the final manuscript.

\section{Funding}

Computed Tomography (CT) angiography and laboratory assessments were financed by Medical Affairs division of Getz Pharma.

\section{Availability of data and materials}

The data for the current study are available on request.

\section{Ethics approval and consent to participate}

The ethical approval for the study was obtained from the Ethical Review Committee of National Institute of Cardio-Vascular Diseases (NICVD), Karachi, Pakistan (ERC-29/2018).

"Study participants were enrolled after obtaining informed written consent and those study participants who were found to have either congenital heart disease or valvular heart diseases or ischemic heart disease were excluded from the study based on initial screening. Individuals who refused or shown reluctance to accept computed tomography angiography (CTA) or other laboratory assessments such as lipid profile or C-reactive protein (hs-CRP) were excluded after an initial assessment".

\section{Consent for publication}

The manuscript does not contain any individual's identifiable information or data.

\section{Competing interests}

The authors declare that there are no competing interests.

\section{Author details}

${ }^{1}$ National Institute of Cardiovascular Diseases (NICVD), Karachi, Pakistan. ${ }^{2}$ Combined Military Hospital (CMH) Malir, Karachi, Pakistan. ${ }^{3}$ Aga Khan University Hospital, Karachi, Pakistan.
Received: 3 December 2019 Accepted: 5 March 2020

Published online: 27 March 2020

\section{References}

1. Barquera S, Pedroza-Tobías A, Medina C, Hernández-Barrera L, BibbinsDomingo K, Lozano R, et al. Global overview of the epidemiology of atherosclerotic cardiovascular disease. Arch Med Res. 2015;46(5):328-38.

2. Cappuccio FP, Miller MA. Cardiovascular disease and hypertension in subSaharan Africa: burden, risk and interventions. Intern Emerg Med. 2016;11(3): 299-305.

3. Lackland DT, Weber MA. Global burden of cardiovascular disease and stroke: hypertension at the core. Can J Cardiol. 2015;31(5):569-71.

4. Roth GA, Johnson C, Abajobir A, Abd-Allah F, Abera SF, Abyu G, et al. Global, regional, and national burden of cardiovascular diseases for 10 causes, 1990 to 2015. J Am Coll Cardiol. 2017:70(1):1-25.

5. Roth GA, Johnson CO, Abate KH, Abd-Allah F, Ahmed M, Alam K, et al. The burden of cardiovascular diseases among US states, 1990-2016. JAMA Cardiol. 2018;3(5):375-89.

6. Volgman AS, Palaniappan LS, Aggarwal NT, Gupta M, Khandelwal A, Krishnan $\mathrm{AV}$, et al. Atherosclerotic cardiovascular disease in south Asians in the United States: epidemiology, risk factors, and treatments: a scientific statement from the American Heart Association. Circulation. 2018;138(1):e1-e34.

7. Goyal A, Yusuf S. The burden of cardiovascular disease in the Indian subcontinent. Indian J Med Res. 2006;124(3):235-44.

8. Khera A, Budoff MJ, O'Donnell CJ, Ayers CA, Locke J, de Lemos JA, et al. Astronaut cardiovascular health and risk modification (Astro-CHARM) coronary calcium atherosclerotic cardiovascular disease risk calculator. Circulation. 2018;138(17):1819-27.

9. Karmali KN, Goff DC, Ning H, Lloyd-Jones DM. A systematic examination of the 2013 ACC/AHA pooled cohort risk assessment tool for atherosclerotic cardiovascular disease. J Am Coll Cardiol. 2014;64(10):959-68.

10. Ashraf T, Achakzai AS, Farooq F, Memon MA, Mengal N, Abbas KY, et al. Estimating risk of atherosclerotic cardiovascular diseases in nonatherosclerotic Pakistani patients: study conducted at National Institute of Cardiovascular Diseases, Karachi, Pakistan. J Pak Med Assoc. 2017;67(April (4)):494-8.

11. Batra MK, Rizvi NH, Sial JA, Saghir T, Karim M. Angiographic characteristics and in hospital outcome of young patients, age up to 40 versus more than 40 years undergoing primary percutaneous coronary intervention. J Pak Med Assoc. 2019;69(4):1307-11.

12. Hassan K, Mohydin B, Fawwad A, Waris N, lqbal S, Jawaid M. Predicting the risk of atherosclerotic cardiovascular disease (ASCVD) in Pakistani population. Clin Epidemiol Glob Health. 2019;7(2):184-7.

13. Ruwanpathirana T, Owen A, Reid CM. Review on cardiovascular risk prediction. Cardiovasc Ther. 2015;33(2):62-70.

14. Bazo-Alvarez JC, Quispe R, Peralta F, Poterico JA, Valle GA, Burroughs M, et al. Agreement between cardiovascular disease risk scores in resourcelimited settings: evidence from 5 Peruvian sites. Crit Pathw Cardiol. 2015; 14(2):74-80.

15. Arnett DK, Blumenthal RS, Albert MA, Buroker AB, Goldberger ZD, Hahn EJ, et al. 2019 ACC/AHA guideline on the primary prevention of cardiovascular disease: a report of the American College of Cardiology/American Heart Association task force on clinical practice guidelines. J Am Coll Cardiol. 2019;74(10):e177-232.

16. Blaha MJ, Cainzos-Achirica M, Greenland P, McEvoy JW, Blankstein R, Budoff $\mathrm{MJ}$, et al. Role of coronary artery calcium score of zero and other negative risk markers for cardiovascular disease: the multi-ethnic study of atherosclerosis (MESA). Circulation. 2016;133(9):849-58.

17. Carr JJ, Jacobs DR, Terry JG, Shay CM, Sidney S, Liu K, et al. Association of coronary artery calcium in adults aged 32 to 46 years with incident coronary heart disease and death. JAMA Cardiol. 2017;2(4):391-9.

18. Cozlea D, Farcas D, Nagy A, Keresztesi A, Tifrea R, Cozlea L, et al. The impact of $C$ reactive protein on global cardiovascular risk on patients with coronary artery disease. Curr Health Sci J. 2013;39(4):225-31.

19. Bilen O, Kamal A, Virani SS. Lipoprotein abnormalities in south Asians and its association with cardiovascular disease: current state and future directions. World J Cardiol. 2016;8(3):247-57.

\section{Publisher's Note}

Springer Nature remains neutral with regard to jurisdictional claims in published maps and institutional affiliations. 